\title{
Comparison of the Hemagglutination Inhibition Test and IgG ELISA in Categorizing Primary and Secondary Dengue Infections Based on the Plaque Reduction Neutralization Test
}

\author{
Nurhayati Lukman, ${ }^{1,2}$ Gustiani Salim, ${ }^{2}$ Herman Kosasih, ${ }^{1,2}$ Nugroho Harry Susanto, ${ }^{2,3}$ \\ Ida Parwati, ${ }^{4}$ Silvita Fitri, ${ }^{3}$ Bachti Alisjahbana, ${ }^{3}$ Susana Widjaja, ${ }^{2}$ and Maya Williams ${ }^{2}$ \\ ${ }^{1}$ Indonesia Research Partnership on Infectious Diseases (INA-RESPOND), Jalan Percetakan Negara No. 29, Jakarta 10560, Indonesia \\ ${ }^{2}$ U.S. Naval Medical Research Unit No. 2, Jakarta 10560, Indonesia \\ ${ }^{3}$ Health Research Unit, Universitas Padjadjaran, Jalan Eijkman No. 38, Bandung 40161, Indonesia \\ ${ }^{4}$ Clinical Pathology Department, Hasan Sadikin Hospital, Faculty of Medicine, Universitas Padjadjaran, \\ Jalan Pasteur No. 38, Bandung 40161, Indonesia
}

Correspondence should be addressed to Herman Kosasih; hermaninarespond@gmail.com

Received 19 January 2016; Revised 9 May 2016; Accepted 26 May 2016

Academic Editor: Peter A. C. Maple

Copyright (C) 2016 Nurhayati Lukman et al. This is an open access article distributed under the Creative Commons Attribution License, which permits unrestricted use, distribution, and reproduction in any medium, provided the original work is properly cited.

Secondary dengue infection by heterotypic serotypes is associated with severe manifestations of disease, that is, dengue hemorrhagic fever (DHF) and dengue shock syndrome (DSS). The World Health Organization (WHO) has recommended criteria based on the hemagglutination inhibition (HI) test to distinguish between primary and secondary dengue infections. Since the HI test has practical limitations and disadvantages, we evaluated the accuracy of WHO HI criteria and compared it with criteria based on an IgG enzyme-linked immunosorbent assay (ELISA) using a plaque reduction neutralization test (PRNT) as the gold standard. Both WHO HI criteria and IgG ELISA criteria performed strongly (16/16) in determining primary infection. However, to determine secondary infection, the IgG ELISA criteria performed better (72/73) compared to the WHO HI criteria (23/73).

\section{Introduction}

Dengue virus is a global concern with an increasing incidence, especially in endemic areas like Southeast Asia, South America, and the Pacific. Recent analysis, based on the geographical distribution of the disease, estimates 250-500 million infections annually, which is three times higher than the estimation from the World Health Organization (WHO) $[1,2]$.

Dengue virus (DENV) belongs to the family Flaviviridae, genus Flavivirus, which consists of four antigenically distinct serotypes (DENV-1, DENV-2, DENV-3, and DENV-4). Dengue virus infection can be confirmed by virus culture, by reverse-transcription polymerase chain reaction (RT-PCR), serologically by neutralization test (gold standard), by IgM enzyme-linked immunosorbent assay (ELISA) antibodies, or by 4 -fold increase of hemagglutination inhibition (HI) antibody titers between acute and convalescent specimens. $\mathrm{HI}$ test is more commonly used than PRNT as HI test does not need virus culture facilities and has a simpler technique [3].

Infection with any of the four serotypes can be asymptomatic or results in a wide range of clinical manifestations between dengue fever (DF), mild to severe dengue hemorrhagic fever (DHF), and dengue shock syndrome (DSS) which is often fatal. Besides viral virulence and host background (immune, genetic, and nutritional), severe dengue infection is often associated with secondary infections [4]. Secondary infections, which are common in endemic areas such as Indonesia [5-8], comprised approximately $80-90 \%$ of the DHF/DSS cases. Therefore, it is important to distinguish between primary and secondary infections. The 2009 WHO 
guidelines on dengue suggested criteria based on HI titers in convalescent sera to distinguish between primary and secondary infection. While the accuracy of the WHO HI criteria in distinguishing between primary and secondary infections has been questioned [9-14], these criteria are still used in studies which analyze the relationship between primary or secondary infections, disease severity, and studies conducted to develop serological methods [9, 10,15-17]. Several methods based on ELISA, which are easier and faster to perform, have been proposed as alternative methods to distinguish between primary and secondary infections, for example, by measuring IgG antibodies; the avidity titer of IgG; calculating the ratio of $\operatorname{IgM} / \operatorname{IgG}$; or the absence of IgG in acute specimens [11, 12, $14,18,19]$. However, none of these studies utilized another serological assay as the gold standard. In this study, we compared the utility of the WHO HI criteria and IgG ELISA in differentiating primary and secondary infections using acute and convalescent specimens that had been tested by a plaque reduction neutralization test (PRNT). In addition, we also propose modified HI criteria to distinguish between primary and secondary infections.

\section{Materials and Methods}

2.1. Sample Collection. This study used 178 acute and convalescent sera from 89 confirmed dengue cases. These sera were collected following written informed consent as a part of two dengue studies in Bandung. These studies were approved by the Institutional Review Board of the United Stated Naval Medical Research Unit (US-NAMRU)\#2 (N2.2006.0001 and N2.2004.0010) in compliance with all US Federal Regulations governing the protection of human subjects and at the National Institute of Health Research and Development, Ministry of Health, Indonesia (KS.02.01.2.1.2776 and KS.02.01.2.1.3461). Study procedures were done in accordance with the Helsinki Declaration of 1975, as revised in 2013.

Acute sera were taken when study participants came to health centers or hospitals, while convalescent sera were obtained at least seven days later or when the participants were discharged from the hospitals.

2.2. Dengue Laboratory Assays. A dengue infection was confirmed when (1) DENV was isolated from tissue culture or DENV RNA was detected by the RT-PCR or (2) when the acute sample was positive for IgM and paired sera demonstrated increasing IgG titers and/or a fourfold rise in HI titers. Primary or secondary infection was confirmed by PRNT, IgG ELISA, and HI tests.

2.2.1. Dengue IgM ELISA Test. Dengue IgM tests were conducted using commercial ELISA kits (Dengue IgM ELISA, Focus Technologies, Cypress, CA, USA). The tests were performed following manufacture's procedures as previously described [20].

2.2.2. Dengue IgG ELISA Test. IgG ELISA was performed using a commercial dengue IgG ELISA kit (Dengue IgG ELISA, Focus Technologies, Cypress, CA, USA), following the manufacturer's instructions. Index value was calculated by dividing sample optical density (OD) with mean of calibrator OD. Specimens with an IgG index value $<1$ were confirmed as negative and those with IgG index value $>1$ were confirmed as positive. Dengue case with negative IgG results from the acute specimen was classified as a primary infection.

2.2.3. Dengue HI Test. HI test was performed according to the procedures [21]. A preliminary experiment comparing the performance of all four dengue antigens in the HI test did not show any difference in their ability determining recent infection or in differentiating primary and secondary infections (data not shown). Hence, only dengue-3 antigen was used for HI test throughout the rest of this study. Specimens with detectable $\mathrm{HI}$ antibodies at $1: 10$ or higher dilutions were confirmed as positive. If no antibody was detected at the lowest dilution $(1: 10)$, then the specimen was confirmed as negative.

WHO recommended using HI titers of convalescent sera as the criteria to distinguish between primary and secondary infection. Dengue infection with convalescent HI antibody titer higher than 1280 was classified as a secondary infection $[1]$.

2.2.4. PRNT. Plaque reduction neutralization test (PRNT) was performed using the following reference strains: DENV1 (16001), DENV-2 (16682), DENV-3 (16562), and DENV-4 (1036). In brief, 500 plaque forming units per milliliter of dengue virus were incubated with serial 4 -fold diluted serum from $1: 10$ to $1: 10,240$. A suspension of the baby hamster kidney (BHK) cells was used to detect the virus reduction following previously described methods [22]. $\mathrm{PRNT}_{50}$ titer was calculated using a log probit regression analysis by SPSS (SPSS Inc., Chicago, IL). Negative result for neutralizing antibodies was confirmed when $\mathrm{PRNT}_{50}$ values were lower than 10 against all dengue serotypes. A positive result for neutralizing antibodies was confirmed when $\mathrm{PRNT}_{50}$ titers were 10 or greater for at least one dengue serotype. Cases with seroconversion of neutralizing antibodies were classified as a primary infection; and cases with detectable neutralizing antibodies in acute specimen were confirmed as a secondary infection.

2.3. Statistical Analysis. Statistical analysis was performed using STATA version 9 (STATA Corporation, TX). Comparison between the two assays was analyzed using McNemar's test.

\section{Results}

Subject demographics and specimen characteristics categorized by primary and secondary infection according to PRNT results are presented in Table 1 . While subjects with a primary infection were slightly younger than those subjects with a secondary infection, there was no significant difference in terms of age, gender distribution, acute sample collection day, convalescent sample collection day, or the interval between acute and convalescent sample collection.

3.1. HI and IgG ELISA Performances Compared to PRNT. First, we sought to examine the sensitivity and specificity of 
TABLE 1: Subject demographics and sample characteristics.

\begin{tabular}{|c|c|c|c|c|c|}
\hline & \multicolumn{2}{|r|}{ Subjects } & \multicolumn{3}{|c|}{ Samples } \\
\hline & Age $\left(\right.$ years $\left.{ }^{*}\right)$ & Male (\%)/female (\%) & Acute (days of onset ${ }^{*}$ ) & Convalescent (days of onset ${ }^{*}$ ) & A-C interval (days*) \\
\hline Primary $(n=16)$ & $26 \pm 9,(7-42)$ & $13(81) / 3(19)$ & $2.5 \pm 1.7,(1-7)$ & $18.5 \pm 5.9,(9-32)$ & $15.9 \pm 5.6,(7-30)$ \\
\hline Secondary $(n=73)$ & $36 \pm 8,(9-53)$ & $47(64) / 26(36)$ & $2.1 \pm 1.3,(1-7)$ & $15.3 \pm 4.4,(6-28)$ & $13.2 \pm 3.9,(4-26)$ \\
\hline Total $(n=89)$ & $34 \pm 9,(7-53)$ & $60(67) / 29(33)$ & $2.2 \pm 1.4,(1-7)$ & $15.9 \pm 4.8,(6-32)$ & $13.7 \pm 4.4,(4-30)$ \\
\hline
\end{tabular}

IgG ELISA and HI test in comparison to PRNT. Based on PRNT results, 162 of 178 specimens were positive for dengue neutralizing antibodies and 16 specimens (all acute) were negative. Among the 162 positive samples, IgG ELISA results were positive for 161 (99.4\%) samples and HI test results were positive for 153 (94.4\%) samples. Among the 16 specimens confirmed negative by PRNT, IgG ELISA results were also negative for these samples, whereas HI test results were negative in only $10(62.5 \%)$ samples. Thus, while the sensitivities between ELISA and HI test were similar compared to PRNT, the IgG ELISA was significantly more specific than the HI test $(p<0.01)$.

\subsection{Distinguishing between Primary and Secondary Infections.} Next, we aimed to determine the performance of IgG ELISA and WHO HI criteria in distinguishing between primary and secondary infection using titer from convalescent samples with PRNT results as the gold standard. According to PRNT results, from 89 dengue cases included in this study, 16 cases were categorized as a primary infection and 73 cases were categorized as a secondary infection. Results from both HI test using convalescent samples and IgG ELISA using acute samples concurred in all of the 16 primary cases. IgG ELISA also identified 72 of $73(98.6 \%)$ secondary cases, whereas HI test was only able to detect 23 of the $73(31.5 \%)$ secondary cases. The difference between the two results was significant $(p<$ 0.01).

In Table 2, our results show that 11 cases with convalescent HI titers $\leq 80$ were all confirmed as a primary infection by PRNT. In 32 cases with convalescent HI titers between 160 and 640 , which are considered as a primary infection by WHO criteria, PRNT results only confirmed five (15.6\%) cases. All 46 cases with convalescent $H I$ titers $\geq 1280$ were also confirmed as a secondary infection by PRNT.

To determine if we could better categorize the cases with convalescent HI titers between 160 and 640, we considered HI results from the acute samples of these cases. Cases with convalescent HI titers between 160 and 640 and negative HI titer in acute samples were considered as a primary infection. Cases with convalescent HI titers between 160 and 640 and positive $\mathrm{HI}$ titers in acute samples were considered as a secondary infection (Table 3, modified HI-1).

Applying this modified classification, 2 of the 5 cases in confirmed primary infection by PRNT did not have HI antibodies in the acute samples, whereas 22 of 27 cases in confirmed secondary infection by PRNT had HI antibodies in the acute specimens. Hence, these 24 cases were classified in concordance with PRNT results. Using this criteria, 13/16 (81.3\%)
TABLE 2: Convalescent HI titers and case classification according to PRNT.

\begin{tabular}{lccccc}
\hline \multirow{2}{*}{ HI titers } & Cases & \multicolumn{5}{c}{ Primary infection } & Secondary infection \\
& & Cases & $\%$ & Cases & $\%$ \\
\hline$\leq 80$ & 11 & 11 & $100.0 \%$ & 0 & $0.0 \%$ \\
160 & 4 & 1 & $25.0 \%$ & 3 & $75.0 \%$ \\
320 & 14 & 3 & $21.4 \%$ & 11 & $78.6 \%$ \\
640 & 14 & 1 & $7.1 \%$ & 13 & $92.9 \%$ \\
1280 & 23 & 0 & $0.0 \%$ & 23 & $100 \%$ \\
$\geq 2560$ & 23 & 0 & $0.0 \%$ & 23 & $100 \%$ \\
\hline
\end{tabular}

primary cases and 68/73 (93.2\%) secondary cases were classified in concordance with PRNT results. We also calculated based on acute sample HI results for classification by defining cases with no $\mathrm{HI}$ antibodies in acute samples as primary infections and cases with $\mathrm{HI}$ antibodies in acute samples as secondary infection (Table 3, modified HI-2). Using this criteria, 10/16 (62.5\%) primary cases and 64/73 (87.7\%) secondary cases were classified in concordance with PRNT results.

Table 3 summarizes the percentage of cases classified in concordance with PRNT results as a primary or a secondary infection using all of the criteria examined in this study. Cases classified according to acute sample IgG ELISA results had the highest concordance with PRNT classification, while criteria that took into account both convalescent and acute HI results had the next best concordance with PRNT classification. The performance in identifying primary infection is statistically different between modified HI-2 and IgG ELISA and modified HI-2 and WHO HI criteria. The performance in identifying secondary infection is statistically different between WHO HI and all other criteria. It is also significantly different between IgG ELISA and modified HI-2.

\section{Discussion}

Serology assays are the most applicable and affordable confirmatory test for dengue infections in third world countries where dengue is commonly endemic. In addition, WHO has recommended IgM Antibody Capture (MAC) ELISA, HI test, and PRNT to determine between primary and secondary infection [21]. Compared to MAC ELISA and PRNT, HI test has been the most widely used serology assay for a long time. However, similar to previous reports, we showed that $\mathrm{HI}$ test underestimated secondary infection when the WHO 
TABLE 3: Percentage of cases classified in concordance with PRNT classification, using IgG ELISA, WHO HI criteria, or modified HI criteria.

\begin{tabular}{lcccc}
\hline & IgG ELISA & WHO HI criteria & Modified HI-1 $^{\mathrm{a}}$ & Modified HI-2 $^{\mathrm{b}}$ \\
\hline Primary infection & $16 / 16(100)$ & $16 / 16(100)$ & $13 / 16(81.25)$ & $10 / 16(62.5)$ \\
Secondary infection & $72 / 73(98.6)$ & $23 / 73(31.5)$ & $68 / 73(93.2)$ & $64 / 73(87.7)$ \\
\hline
\end{tabular}

${ }^{a}$ Primary infection if convalescent HI antibody titers are $\leq 80$ or if convalescent $\mathrm{HI}$ antibody titers are 160,320 , or 640 and HI antibody titers negative in acute specimen; ${ }^{b}$ primary infection if $\mathrm{HI}$ antibody titers are negative in acute specimen.

recommended cutoff was applied $[13,14]$. The low performance of the HI test in secondary infection has also been reported previously by other groups. The HI antibodies do not raise to reach the titer of $1: 1280$, as required by the WHO HI criteria [14]. This might be associated with the degradation of a fraction of $\mathrm{HI}$ antibodies which is below the test detection threshold [13]. By applying modified cutoff criteria with both convalescent and acute sample HI results, the accuracy in determining secondary infection increased from $31.5 \%$ to $93.2 \%$, while the accuracy in determining primary infection decreased from $100 \%$ to $81.3 \%$.

Nevertheless, compared to the HI test, the commercially available ELISA kit is simpler and faster than the HI test. Thus ELISA kit is easier to obtain than $\mathrm{HI}$ antigens in some countries such as Indonesia. Although several types of ELISA evaluation (avidity, ratio $\operatorname{IgM} / \mathrm{IgG}$ ) have been compared with $\mathrm{HI}$ and have been determined to be more reliable than HI tests, these studies did not use a third assay as a reference $[12,14,18$, 19]. By using PRNT as the gold standard, our study has confirmed results from previous studies that ELISA is superior to $\mathrm{HI}$ in determining primary and secondary infections.

Our study was similar to de Souza et al. in regard to the IgG ELISA kit used (Focus Diagnostics) and the simple criteria for determining between primary and secondary infection-the absence or presence of IgG antibodies in the acute specimens [19]. As other IgG ELISA kits may have different sensitivities and specificities, these criteria should only be used after the sensitivity and specificity of these kits are assessed. Compared to other methods that used IgG ELISA and showed similar performance, such as IgM/IgG ratio and avidity index, our method is simpler and more cost-effective.

\section{Conclusion}

Considering the limitation of HI criteria in differentiating between primary and secondary infection, we recommend the use of the absence or presence of IgG antibodies in acute specimens as measured by IgG ELISA as the criteria. HI may still be used; however, the criteria for interpretation should be revised. We propose that $\mathrm{HI}$ convalescent titers $\leq 80$ should be classified as primary infections, HI convalescent titers $\geq 1280$ should be classified as secondary infections, and, for cases with HI convalescent titers between 160 and 640, the acute samples should be evaluated. Cases with acute sample that does not have any detectable HI antibodies should be classified as a primary infection, while cases with acute sample that have detectable $\mathrm{HI}$ antibodies should be classified as a secondary infection. This study shows that performance of acute IgG ELISA criteria and the proposed HI criteria is equally good.

\section{Disclosure}

The sponsor had no involvement in the study design, the collection, analysis, and interpretation of data, the writing of the report, or the decision to submit the paper for publication. All authors have read and approved the final version of the paper. The views expressed in this paper are those of the authors and do not necessarily reflect the official policy or position of the Department of the Navy, Department of Defense, or the US Government. Some of the authors are military service members or employees of the US Government. This work was prepared as part of their official duties. Title 17 U.S.C. $\$ 105$ provides that "Copyright protection under this title is not available for any work of the United States Government." Title 17 U.S.C. $\$ 101$ defines a US Government work as a work prepared by a military service member or employee of the US Government as part of that person's official duties.

\section{Competing Interests}

All the authors declare they have no conflict of interests.

\section{Acknowledgments}

The authors would like to thank INA-RESPOND for all the support during the preparation of this paper and all the volunteers, dengue research teams at the factories and Hasan Sadikin Hospital, National Institute Health Research and Development, Ministry of Health, Indonesia, and NAMRU\#2 for their dedication in dengue research. They would also like to thank Kendra Chittenden from the USAID and Aly Diana from INA-RESPOND for their support and assistance.

\section{References}

[1] World Health Organization, Dengue Haemorrhagic Fever: Diagnosis, Treatment, Prevention, and Control, World Health Organization, Geneva, Switzerland, 2009.

[2] S. Bhatt, P. W. Gething, O. J. Brady et al., "The global distribution and burden of dengue," Nature, vol. 496, no. 7446, pp. 504-507, 2013.

[3] C.-L. Kao, C.-C. King, D.-Y. Chao, H.-L. Wu, and G.-J. J. Chang, "Laboratory diagnosis of dengue virus infection: current and future perspectives in clinical diagnosis and public health," Journal of Microbiology, Immunology and Infection, vol. 38, no. 1, pp. 5-16, 2005.

[4] B. Guy and J. W. Almond, "Towards a dengue vaccine: progress to date and remaining challenges," Comparative Immunology, Microbiology and Infectious Diseases, vol. 31, no. 2-3, pp. 239252, 2008.

[5] A. Suwandono, H. Kosasih, Nurhayati et al., "Four dengue virus serotypes found circulating during an outbreak of dengue fever 
and dengue haemorrhagic fever in Jakarta, Indonesia, during 2004," Transactions of the Royal Society of Tropical Medicine and Hygiene, vol. 100, no. 9, pp. 855-862, 2006.

[6] S.-S. Sam, S. F. S. Omar, B.-T. Teoh, J. Abd-Jamil, and S. AbuBakar, "Review of dengue hemorrhagic fever fatal cases seen among adults: a retrospective Study," PLoS Neglected Tropical Diseases, vol. 7, no. 5, Article ID e2194, 2013.

[7] M. M. Ngwe Tun, K. Z. Thant, S. Inoue et al., "Serological characterization of dengue virus infections observed among dengue hemorrhagic fever/dengue shock syndrome cases in upper Myanmar," Journal of Medical Virology, vol. 85, no. 7, pp. 12581266, 2013.

[8] T. M. Sharp, E. Hunsperger, G. A. Santiago et al., "Virus-specific differences in rates of disease during the 2010 dengue epidemic in Puerto Rico," PLoS Neglected Tropical Diseases, vol. 7, no. 4, Article ID e2159, 2013.

[9] M. P. Miagostovich, R. M. R. Nogueira, F. B. dos Santos, H. G. Schatzmayr, E. S. M. Araújo, and V. Vorndam, "Evaluation of an IgG enzyme-linked immunosorbent assay for dengue diagnosis," Journal of Clinical Virology, vol. 14, no. 3, pp. 183-189, 1999.

[10] P.-Y. Shu, L.-K. Chen, S.-F. Chang et al., "Comparison of capture immunoglobulin $\mathrm{M}$ (IgM) and IgG enzyme-linked immunosorbent assay (ELISA) and nonstructural protein NS1 serotypespecific IgG ELISA for differentiation of primary and secondary dengue virus infections," Clinical and Diagnostic Laboratory Immunology, vol. 10, no. 4, pp. 622-630, 2003.

[11] S. Matheus, X. Deparis, B. Labeau, J. Lelarge, J. Morvan, and P. Dussart, "Use of four dengue virus antigens for determination of dengue immune status by enzyme-linked immunosorbent assay of immunoglobulin G avidity," Journal of Clinical Microbiology, vol. 43, no. 11, pp. 5784-5786, 2005.

[12] S. Matheus, X. Deparis, B. Labeau, J. Lelarge, J. Morvan, and P. Dussart, "Discrimination between primary and secondary dengue virus infection by an immunoglobulin $G$ avidity test using a single acute-phase serum sample," Journal of Clinical Microbiology, vol. 43, no. 6, pp. 2793-2797, 2005.

[13] R. R. Graham, M. Juffrie, R. Tan et al., "A prospective seroepidemiologic study on dengue in children four to nine years of age in Yogyakarta, Indonesia I. Studies in 1995-1996," The American Journal of Tropical Medicine and Hygiene, vol. 61, no. 3, pp. 412419, 1999.

[14] A. Atchareeya, P. Songthum, S. Areerat et al., "Comparison between haemagglutination inhibition (HI) test and IgM and IgG-capture ELISA in determination of primary and secondary dengue virus infections," Dengue Bulletin, vol. 30, pp. 141-145, 2006.

[15] S. K. Lam and P. L. Devine, "Evaluation of capture ELISA and rapid immunochromatographic test for the determination of IgM and IgG antibodies produced during dengue infection," Clinical and Diagnostic Virology, vol. 10, no. 1, pp. 75-81, 1998.

[16] A. Becerra, R. V. Warke, N. de Bosch, A. L. Rothman, and I. Bosch, "Elevated levels of soluble ST2 protein in dengue virus infected patients," Cytokine, vol. 41, no. 2, pp. 114-120, 2008.

[17] M. Sriprom, P. Pongsumpun, S. Yoksan, P. Barbazan, J. P. Gonzalez, and I. M. Tang, "Dengue haemorrhagic fever in Thailand, 1998-2003: primary or secondary infection," Dengue Bulletin, vol. 27, pp. 39-45, 2003.

[18] D. W. Vaughn, A. Nisalak, T. Solomon et al., "Rapid serologic diagnosis of dengue virus infection using a commercial capture ELISA that distinguishes primary and secondary infections," The American Journal of Tropical Medicine and Hygiene, vol. 60, no. 4, pp. 693-698, 1999.
[19] V. A. U. F. de Souza, A. F. Tateno, R. R. Oliveira et al., "Sensitivity and specificity of three ELISA-based assays for discriminating primary from secondary acute dengue virus infection," Journal of Clinical Virology, vol. 39, no. 3, pp. 230-233, 2007.

[20] K. R. Porter, S. Widjaja, H. D. Lohita et al., "Evaluation of a commercially available immunoglobulin $\mathrm{M}$ capture enzymelinked immunosorbent assay kit for diagnosing acute dengue infections," Clinical and Diagnostic Laboratory Immunology, vol. 6, no. 5, pp. 741-744, 1999.

[21] D. H. Clarke and J. Casal, "Technique for hemagglutination and hemagglutination inhibition with arthropod borne virus," The American Journal of Tropical Medicine and Hygiene, vol. 7, no. 5, pp. 561-567, 1958.

[22] D. M. Morens, S. B. Halstead, P. M. Repik, R. Putvatana, and N. Raybourne, "Simplified plaque reduction neutralization assay for dengue viruses by semimicro methods in BHK-21 cells: comparison of the BHK suspension test with standard plaque reduction neutralization," Journal of Clinical Microbiology, vol. 22, no. 2, pp. 250-254, 1985. 


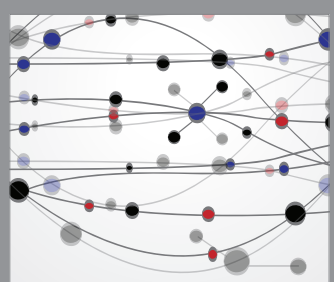

The Scientific World Journal
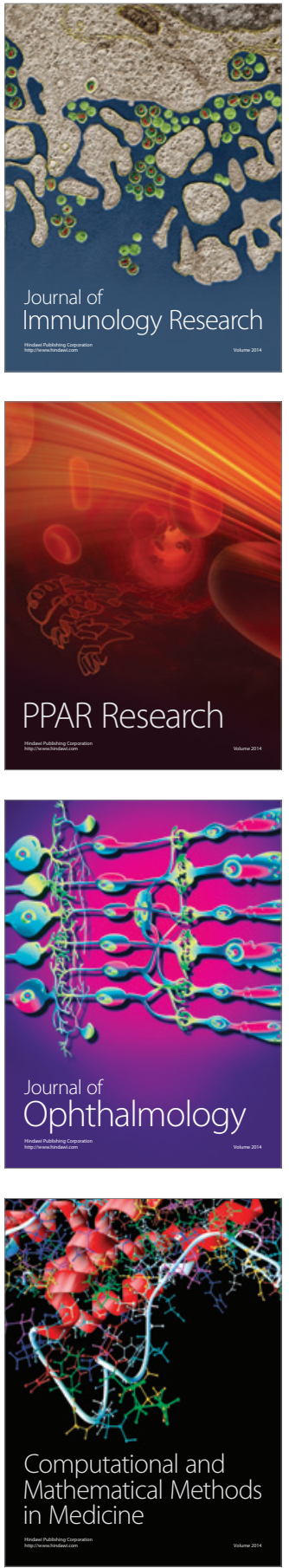

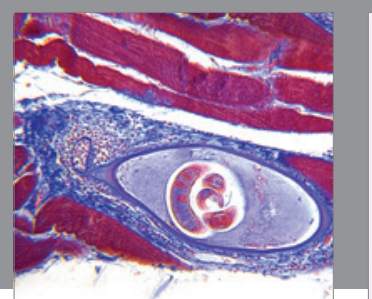

Gastroenterology Research and Practice

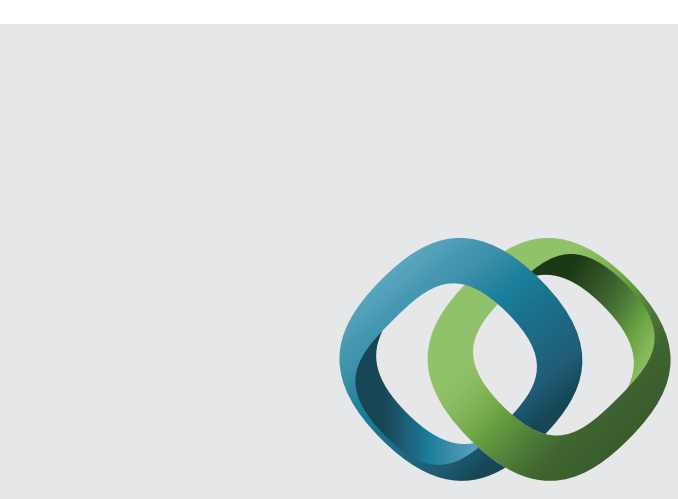

\section{Hindawi}

Submit your manuscripts at

http://www.hindawi.com
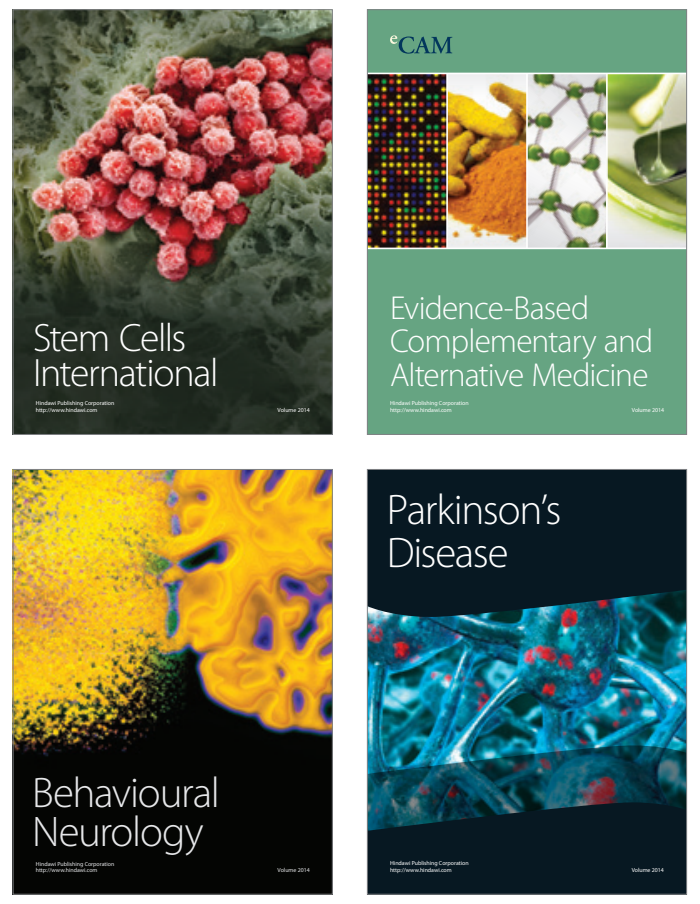
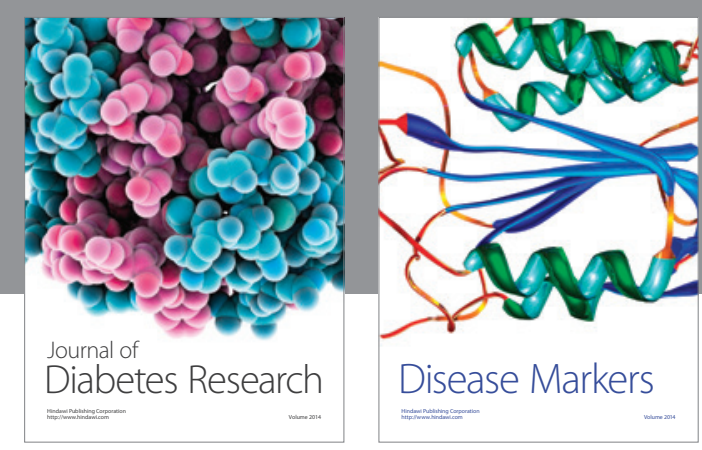

Disease Markers
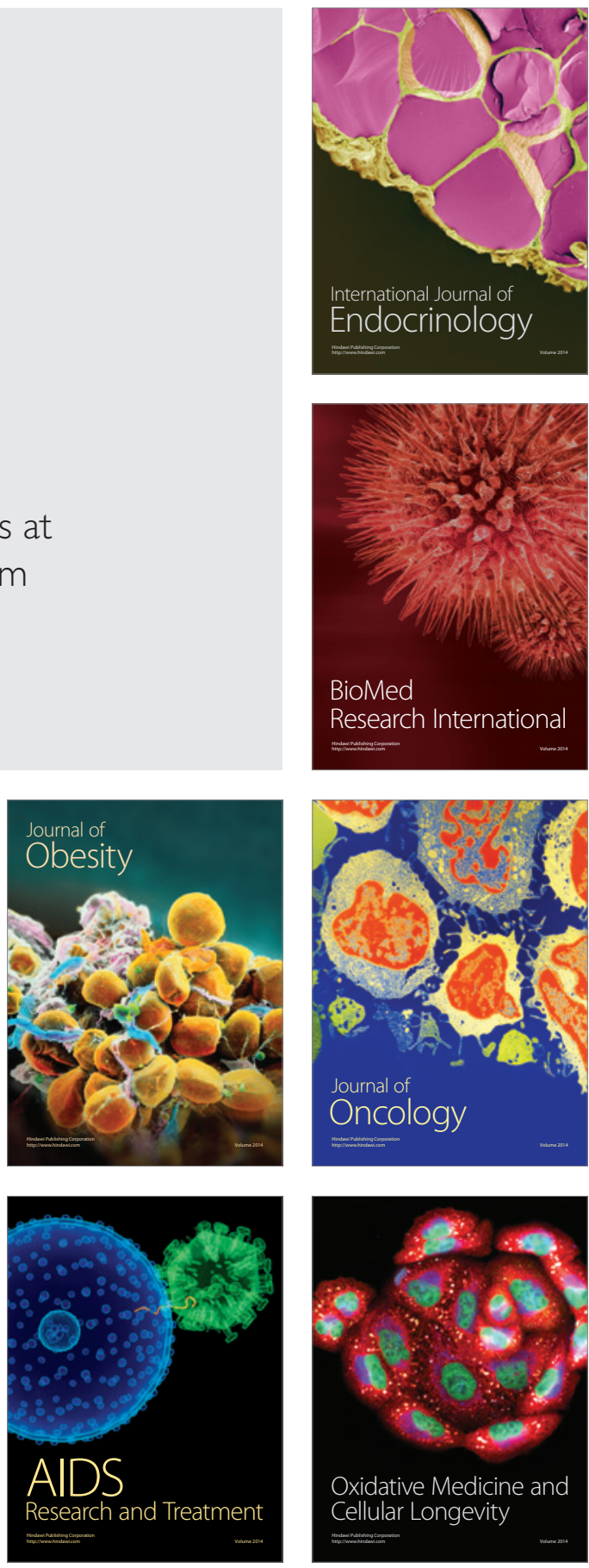